\title{
Panorama de la coinfección tuberculosis/VIH en Bogotá, 2001
}

\author{
Ingrid García ${ }^{1}$, Adriana Merchán ${ }^{2}$, Pablo Enrique Chaparro ${ }^{1,3}$, Lilia Edith López ${ }^{4}$ \\ ${ }^{1}$ Centros Control de Enfermedades, Subdirección de Epidemiología y LNR, Instituto Nacional de Salud, \\ Bogotá, D.C., Colombia. \\ 2 Universidad Colegio Mayor de Cundinamarca, Bogotá, D.C., Colombia. \\ ${ }^{3}$ Estadísticas Vitales, Dirección de Censos y Demografía, Departamento Administrativo Nacional de \\ Estadística, Bogotá, D.C., Colombia. \\ ${ }^{4}$ Programa de Tuberculosis, Secretaría Distrital de Salud de Bogotá, Bogotá, D.C., Colombia.
}

Este estudio pretende describir la situación de los casos de coinfección tuberculosis-virus de inmunodficiencia humano (TB-VIH) reportados en Bogotá en el 2001. Se realizó un estudio descriptivo transversal de los casos con coinfección TB-VIH, reportados por la Secretaría Distrital de Salud de Bogotá durante el 2001, basado en los casos diagnosticados de TB que presentaron prueba positiva para VIH. Se registraron 113 casos $(11,9 \%)$ con coinfección TB-VIH/sida y se diligenciaron 103 tarjetas de control de tratamiento. La mayor proporción de enfermos correspondió al sexo masculino (92,2\%), con una razón hombre: mujer de 12 : 1; 38,8\% de los casos se encontraba entre los 25 y los 34 años. El 40,8\% de los pacientes pertenecía al régimen contributivo de aseguramiento en salud; $81,6 \%$, casos nuevos; $61,9 \%$ de los casos fueron pulmonares y $29,7 \%$ extrapulmonares. De los extrapulmonares, la tuberculosis ganglionar fue la más frecuente. De 55 pacientes con criterio diagnóstico, en $78 \%$ se confirmó la realización de baciloscopia. En 29 casos se registraron los tratamientos utilizados en la primera fase; de éstos, $86,2 \%$ siguió el esquema normado. Las fallas evidenciadas en el sistema de información no permiten conocer completamente el seguimiento de los pacientes y muestran la necesidad de fortalecer el programa de control de la tuberculosis.

Palabras clave: tuberculosis/complicaciones, VIH, coinfección.

Overview of the HIV/Tuberculosis coinfection in Bogotá, Colombia, 2001.

The magnitude and characteristics of the co-infection of TB-AIDS was evaluated in cases reported in Bogotá, 2001. A cross sectional study was carried out using the epidemiological registry of TBC-AIDS cases from the Health Secretariat of Bogotá. Only TB cases with a positive HIV test were included in. Of 950 TB cases reported in Bogotá, 113 (11.9\%) were indicated to have TB and HIV positive tests; epidemiological information was recovered from 103 of them. Most cases were male (male:female ratio was $12: 1$ ); 38.8\% were between ages 25 to $34 ; 40.8 \%$ of cases belonged to the contributive regime; $82 \%$ were new cases. Of the 105 cases, $62 \%$ were classified with the pulmonary form of TB and $30 \%$ with extra pulmonary disease. In the last group, the most frequent form was lymph node tuberculosis. Diagnostic criteria were recorded for only 55 patients; however,78\% were confirmed with microscopic smears.. Procedural data were available for 29 patients in the first phase of treatments; $86.2 \%$ had received therapy recommended by the Ministry of Health. The failures of TB control programs indicated by statistical data, do not reflect patient's activities that may be contributing to greater infection risk. Additional monitoring may be necessary to fortify the current TB control program.

Key words: tuberculosis/complications, HIV infections/etiology. 
La tuberculosis es un problema de salud pública mundial, con 8 millones, aproximadamente, de casos nuevos diagnosticados y 2 millones de muertes cada año (1). La asociación con el virus de la inmunodeficiencia humana $(\mathrm{VIH})$ es un factor de riesgo que agrava la situación epidemiológica de la tuberculosis (2). EI VIH favorece la reactivación de la tuberculosis en personas con infección latente así como la progresión hacia la enfermedad diseminada en aquéllas con primoinfección o reinfección, riesgo que es casi 300 veces mayor que en la población en general (3). La infección por el VIH puede tener una repercusión sobre el comportamiento epidemiológico de la tuberculosis que depende de la prevalencia de la infección con el VIH, la prevalencia de la infección tuberculosa, el riesgo de infección en los sujetos entre los 15 y los 49 años, el patrón de transmisión de la infección por el VIH y el riesgo de los infectados simultáneamente por el VIH y por Mycobacterium tuberculosis de sufrir tuberculosis (4).

Según las cifras de la Organización Mundial de la Salud (OMS), a finales del 2002, se estimaba que había 42 millones de personas con $\mathrm{VIH} /$ sida y una tercera parte de ellos tenía coinfección con tuberculosis. Aproximadamente, $90 \%$ morirá si no reciben el tratamiento adecuado $(5,6)$.

La actual prevalencia de la coinfección de TBVIH en Latinoamérica no se conoce exactamente, aunque se ha estimado que la prevalencia de VIH en los pacientes diagnosticados con tuberculosis es de $5,9 \%$ y que las muertes por tuberculosis atribuibles al VIH se han estimado en 6,5\% (7). Es de resaltar que la alta prevalencia de $\mathrm{VIH} /$ sida en los países del Caribe muestra una vulnerabilidad significativa a la coinfección de TB-VIH. Aunado a lo anterior, la falla en la implementación - la baja cobertura de la población con la estrategia DOTS, hace que falten mecanismos apropiados para el manejo de la tuberculosis y

\footnotetext{
Correspondencia:

Ingrid García, Avenida calle 26 No.51-60, Bogotá, D.C., Colombia.

Teléfono: 220 7700, extensión 150 o 111, fax: 2200934. igarcia@ins.gov.co o ingave@yahoo.com
}

Recibido: 22/08/03; aceptado: 10/02/04 que puede contribuir al aumento de las tasas de coinfección de TB-VIH y, al mismo tiempo, al aumento de las tasas de mortalidad y morbilidad por esta asociación (8).

Los estudios realizados en Colombia han mostrado que la frecuencia de infección por M. tuberculosis en pacientes con VIH fluctúa entre $8 \%$ y $30 \%$; la tuberculosis extrapulmonar tiene un porcentaje importante de estos casos. La frecuencia de coinfección en pacientes con síntomas respiratorios se encuentra alrededor del $20 \%$ (9).

Este estudio pretende describir la situación de los casos de asociación tuberculosis- $\mathrm{VIH} /$ sida reportados en Bogotá durante el 2001, a partir del análisis de las tarjetas de control del tratamiento.

\section{Materiales y métodos}

El sistema de control de la tuberculosis en Bogotá está constituido por entidades públicas y privadas prestadoras de los servicios de salud de primero, segundo y tercer nivel de atención. Las diferentes instituciones deben enviar semanalmente la información al primer nivel de atención para que éstos los informen trimestralmente a la Secretaría Distrital de Salud.

Se realizó un estudio descriptivo de los casos con coinfección de $\mathrm{TB} / \mathrm{VIH}$ reportados a la Secretaría Distrital de Salud de Bogotá en el 2001; se utilizaron las tarjetas individuales de control del tratamiento de cada uno de los pacientes en las diferentes instituciones de salud del Distrito como la fuente de información (10). La tarjeta individual es el registro dinámico y funcional que permite la identificación del enfermo y sus contactos, el estado bacteriológico, la evolución y la intolerancia a los medicamentos; permite conocer el organismo responsable del control, la afiliación al Sistema de Seguridad Social (subsidiado, contributivo, no afiliado o vinculado, otro), el número de enfermos en tratamiento, el cumplimiento en la asistencia a la toma de los medicamentos, la pérdida de control y los resultados del tratamiento de la cohorte.

En los casos incluidos en este estudio, no se determinó cuál de las dos patologías se diagnosticó primero; se trabajó de la misma manera con la información de los casos de 
tuberculosis del Programa de Control de Tuberculosis (PCT) del distrito.

Las definiciones de caso corresponden a las descritas en los protocolos de vigilancia de salud pública de la Secretaría Distrital de Salud de Bogotá. Como 'caso probable de tuberculosis pulmonar' se considera toda persona que presenta tos y expectoración de más de 15 días de duración, y 'caso probable de tuberculosis extrapulmonar', a los pacientes con sintomatología crónica de algunos órganos o sistemas. El caso se confirma como tuberculosis pulmonar si presenta baciloscopia positiva o cultivo positivo, y para tuberculosis extrapulmonar con histopatología, baciloscopia o cultivo positivo para Mycobacterium (11).

Se considera como 'caso probable de infección por VIH' en mayores de 13 años a toda persona con sospecha diagnóstica (presuntiva o confirmada) de enfermedades asociadas con la infección por VIH y sida; antecedentes de relaciones sexuales con un caso confirmado por laboratorio (contacto); antecedentes de haber recibido trasplante o transfusión de paciente o unidad de sangre con confirmación de presencia de VIH por laboratorio, y otros signos y síntomas. El caso en mayores de 18 meses y adultos se considera confirmado para infección por VIH cuando presenta dos pruebas presuntivas inmunoenzimáticas (EIA) reactivas para anticuerpos contra VIH y Western blot o inmunofluorescencia indirecta (IFI) reactivos, y prueba de PCR cualitativa positiva (12).

En el 2001, la información para este estudio provino de 711 unidades notificadoras. Durante ese año, el porcentaje de cumplimiento de la notificación semanal fue de 93,7\% (13).

Se diseñó una base de datos en Epiinfo, versión $6.04 \mathrm{~d}$. Todas las variables se describieron usando proporciones, frecuencias absolutas y relativas. Se calcularon prevalencias con sus intervalos de confianza (IC) del 95\%.

\section{Resultados}

EI PCT de la Secretaría Distrital de Salud de Bogotá reportó 949 casos nuevos de tuberculosis de todas las formas durante el 2001, para una incidencia de 14,4 por 100.000 habitantes; de estos casos, $11,9 \%$ (113 casos) correspondió a la coinfección TB/VIH. De los casos de coinfección, se recibieron en el PCT del distrito el $91,1 \%(103 / 113)$ de las tarjetas individuales de control de la tuberculosis, con un diligenciamiento incompleto.

La mayor proporción de los enfermos con coinfección fue del sexo masculino $(92,2 \%)$, con una razón hombre:mujer de 12:1. La mediana de la edad fue de 34 años; el $38,8 \%$ de los casos se encontraba entre los 25 y los 34 años, y en el $7,8 \%$ de los casos no se consignó la edad (figura 1).

El $40,8 \%$ de los pacientes con el dato de afiliación al sistema de salud pertenecía al régimen contributivo del sistema de aseguramiento en salud. La condición del paciente en el momento de iniciar el tratamiento se consignó en el $91,3 \%$ de los casos; de ellos, el 81,6\% (84 casos) eran nuevos, es decir, pacientes que no habían recibido anteriormente tratamiento para tuberculosis.

En $53 \%$ de los casos con reporte de criterio diagnóstico, se observó que la baciloscopia era positiva en $61,8 \%$ (34 casos). En 16,4\% (9 casos) de los casos que tenían baciloscopia negativa, el diagnóstico se realizó mediante el cultivo y las radiografías de tórax; en $21,8 \%$ (12 casos) no se registró la realización de baciloscopias, el criterio diagnóstico utilizado para determinar que se trataba de tuberculosis se basó en el cultivo y las radiografías de tórax (cuadro 1).

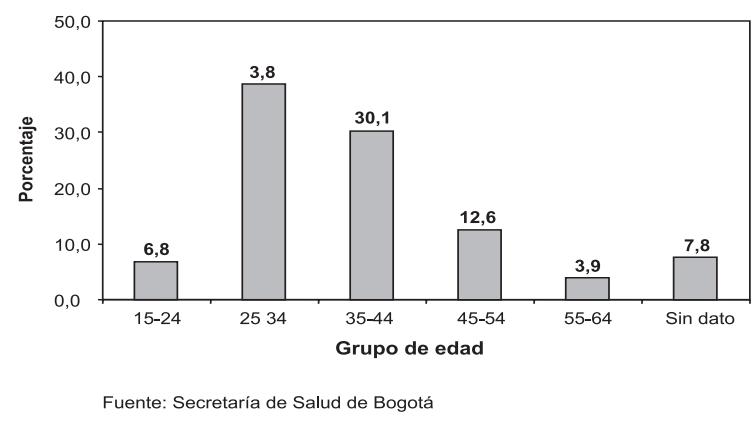

Figura 1. Casos de coinfección TB-VIH, según edad, Bogotá, 2001. 
Cuadro 1. Criterio diagnóstico de tuberculosis en la coinfección con VIH. Bogotá, 2001

\begin{tabular}{|c|c|c|c|c|c|}
\hline \multirow{2}{*}{$\begin{array}{l}\text { Criterio diagnóstico } \\
\text { BK }\end{array}$} & & \multicolumn{2}{|c|}{ Casos tuberculosis } & \multirow[t]{2}{*}{ Total } & \multirow[b]{2}{*}{$\%$} \\
\hline & & $\mathbf{n}$ & $\%$ & & \\
\hline \multirow[t]{3}{*}{ BK positivo } & + & 11 & 20 & & \\
\hline & ++ & 11 & 20 & 34 & 61,8 \\
\hline & +++ & 12 & 21,8 & & \\
\hline Criterio diagnostico & Otro & \multicolumn{2}{|c|}{ Casos tuberculosis } & Total & \\
\hline \multirow[t]{3}{*}{ BK negativo } & Cultivo $(+)$ & 4 & 7,3 & & \\
\hline & Rayos X & 4 & 7,3 & 9 & 16,4 \\
\hline & PPD & 1 & 1,8 & & \\
\hline Sin BKo & Cultivo $(+)$ & 2 & 3,6 & & \\
\hline \multirow[t]{3}{*}{ sin dato de BK } & Rayos X & 5 & 9,1 & 12 & 21,8 \\
\hline & PPD & 1 & 1,8 & & \\
\hline & Sin dato & 4 & 7,3 & & \\
\hline Total & & & & 55 & 100 \\
\hline
\end{tabular}

Fuente: Secretaria Distrital de Salud de Bogotá

Baciloscopia negativa = No se encuentran bacilos ácido alcohol resistente (BAAR) en más de 100 campos microscópicos observados

Baciloscopia positiva $+=$ Se encuentra menos de un BAAR por campo en 100 campos microscópicos observados. Baciloscopia positiva ++ = Se encuentran de 1 a 10 BAAR por campo en 50 campos microscópicos observados.

Baciloscopia positiva +++ = Se encuentran más de 10 BAAR por campo en 20 campos microscópicos observados.

El 53,3\% de los casos reportados con coinfección correspondió a tuberculosis pulmonar y $42,7 \%$ a tuberculosis extrapulmonar; de esta última, la más frecuente fue la tuberculosis ganglionar, seguida de la tuberculosis meníngea y en $3,8 \%$ de los casos se desconoció el tipo de tuberculosis. Se pudo evidenciar un mayor riesgo de tuberculosis extrapulmonar entre los pacientes con reporte de coinfección comparado con las personas con tuberculosis pulmonar (razón de prevalencia $=1,78$, IC 95\% 1,24-2,56).

En 53 casos de coinfección TB-VIH/sida, se consignó la fecha de diagnóstico de la tuberculosis y el inicio de la primera fase de tratamiento. En el $52,8 \%$ de estos pacientes, se inició el tratamiento el mismo día en que se dictaminó la enfermedad, en $20,8 \%$ al día siguiente y en $26,4 \%$ entre el segundo día y 252 días después de su diagnóstico. Al relacionar el hecho de iniciar el tratamiento el mismo día del diagnóstico o uno o varios días posteriores al diagnóstico con el estar afiliado al sistema de salud, se observó que existe un mayor riesgo de iniciar el tratamiento el mismo día del diagnóstico si no se está afiliado con respecto a la población afiliada, aunque esta asociación no es estadísticamente significativa ( $\mathrm{RP}=3,9 ; \mathrm{IC} 95 \%$ 0,88-17,31).
De los pacientes con fechas de inicio de tratamiento de 9 meses o más, solamente en 1 caso $(0,9 \%)$ se hizo el reporte de 'terminó el tratamiento'. El reporte de fallecidos en todas las fichas fue de 16 pacientes (15,5\%).

Las edades de los pacientes reportados como 'muerte' oscilaron entre los 30 y los 50 años con una mediana de 34 años, el tipo de tuberculosis fue pulmonar en $68,8 \%$ de los casos; la baciloscopia fue positiva en $62,5 \%$ de los casos; $81,3 \%$ ingresaron como nuevos al programa y el esquema de tratamiento se reportó en $50 \%$ de los pacientes, siendo este tratamiento el normado a nivel nacional en el $37,5 \%$ del total de casos letales. No se encontraron diferencias de estas variables entre estos pacientes al compararlos con los pacientes que no murieron. En 25\% (4 casos) fue posible determinar el tiempo entre el diagnóstico y la muerte, el cual osciló entre 5 y 25 días con una mediana de 9,5 días.

Con relación a los pacientes que tenían información de tratamiento, el $45,2 \%$ de los casos reportados con coinfección siguió el esquema normado por la Guía de atención de tuberculosis pulmonar y extrapulmonar del Ministerio de Salud, mientras que $7,5 \%$ de los casos se manejaron con esquemas diferentes. 


\section{Discusión}

Una de las limitaciones de este estudio fue la falta parcial de la información contenida en las tarjetas individuales de control del tratamiento; sin embargo, ésta es la única fuente de información del seguimiento de este tipo de pacientes $y$, a pesar de esto, los resultados obtenidos son de gran utilidad para el mejoramiento del programa de control de la tuberculosis.

Existen diferencias metodológicas entre las anteriores investigaciones realizadas en el Distrito Capital $(9,14)$ y el actual estudio, si se tiene en cuenta que se partió de la tarjeta de ingreso al PCT y no fue posible establecer en nuestros resultados la fecha de diagnóstico para cada una de las infecciones.

En Colombia, según el Artículo 37 del Decreto 1543 del 12 de junio de 1997, no se tiene reglamentada la realización del examen de $\mathrm{VIH}$ a los pacientes diagnosticados con tuberculosis; este procedimiento no es obligatorio y, por tanto, está sujeto a la voluntad y aceptación del paciente; de tal forma que para realizar la vigilancia del VIH en los pacientes con tuberculosis se deben utilizar estrategias en las que se incluya el darle a los pacientes la asesoría preprueba y con ello la posibilidad de realizar este diagnóstico.

La razón hombre:mujer de 12:1 en los casos de coinfección TB-VIH encontrada en este estudio es mayor a la reportada por estudios previos hechos en Bogotá, en los cuales la razón hombre: mujer osciló entre $3: 1$ y $5: 1$, similar al realizado en Perú en el 2000 que mostró una razón hombre:mujer de 4:1 (3). Estas diferencias en Bogotá pueden corresponder en gran parte a que los casos nuevos de VIH siguen siendo en hombres que tienen sexo con hombres.

Al ser la tuberculosis y el $\mathrm{VIH} /$ sida enfermedades de interés en salud pública, son alarmantes las graves fallas evidenciadas en el sistema de información que, como consecuencia, llevan a un inadecuado seguimiento de los casos y desconocimiento del estado final del paciente e indirectamente, estarían mostrando irregularidades en el acceso y atención en los servicios de salud.
La letalidad dentro de la población reportada con coinfección tuberculosis-VIH se debió probablemente al diagnóstico tardío de la tuberculosis pulmonar, a las formas diseminadas de la enfermedad o a la inmunodepresión del paciente. Es preocupante el corto tiempo entre la fecha del diagnóstico y la muerte (9,5 días), lo que hace pensar en lo tardío del diagnostico de estos pacientes.

Es importante fomentar la notificación de los casos que se diagnostiquen, la calidad de los registros y llevar el sistema de vigilancia epidemiológica activa, en el cual se comparan y analizan los registros de tuberculosis y de $\mathrm{VIH} /$ sida, con el fin de tomar oportunamente las medidas de intervención.

La determinación de la demora diagnóstica y su origen deben ser minimizadas ya que es un tiempo en el cual el paciente está contagiando a la comunidad y, en el caso de la coinfección, aumenta la probabilidad de muerte del paciente. Para evitar estos problemas, es importante la información y la educación del personal de salud y del público en general sobre los síntomas y signos de la tuberculosis y el $\mathrm{VIH} /$ sida, junto con la atención en salud para todos los enfermos.

\section{Agradecimientos}

Al personal del Programa de Control de la Tuberculosis de la Secretaría Distrital de Salud de Bogotá por la colaboración prestada en la realización del presente estudio.

\section{Referencias}

1. Organización Mundial de la Salud. Global tuberculosis control: OMS Report 2002. Geneva: OMS, Communicable Diseases; 2002.

2. Organización Mundial de la Salud. TB/VIH. Manual clínico para América Latina. 96.200 (S). Ginebra: Organización Mundial de la Salud; 1997.

3. Ministerio de Salud del Perú. Seminario taller: tuberculosis en el Perú. Informe. Situación de la asociación VIH/SIDA-TB. Lima;2000:89-96.

4. Caminero J. Guía de la tuberculosis para médicos especialistas. París, Francia: UICTER; 2003.

5. World Health Organization. WHO report documents worsening global TB/HIV crisis. Medical Letter on the CDC \& FDA. August 5, 2003. 
6. World Health Organization. Guidelines for implementing collaborative TB and HIV programme activities. Italy: WHO, Departament HIV/AIDS; 2003.

7. Corbett E, Watt C, Walker N, Maher D, Williams B, Raviglioni M, Dye C. The growing burden of tuberculosis: global trends and interactions with the VIH epidemic. Arch Intern Med 2003;163:1009-21.

8. Patz D. Construyendo paso a paso: guía sobre atención integral de personas que viven con la coinfección de TB/VIH en América Latina y el Caribe. Documento de trabajo. Washington, D.C.: Organización Panamericana de la Salud; 2003.

9. Yáñez W, Campo A, De la Hoz F, León C, Guerrero MI. Comportamiento epidemiológico de la tuberculosis y el sida, Colombia, 1981-1995. Inf Quinc Epidem Nac 1997;2:74-8.
10. Ministerio de Salud de Colombia. Resolución 412. Guía de atención integral de la tuberculosis pulmonar y extrapulmonar, febrero de 2000.

11. Secretaría Distrital de Salud de Bogotá, D.C. Protocolos de vigilancia de la salud pública: tuberculosis. Bogotá: Secretaría Disrital de Salud; 2001.

12. Secretaría Distrital de Salud de Bogotá, D.C. Protocolos de vigilancia de la salud pública: VIH-sida. Bogotá: Secretaría Disrital de Salud; 2001.

13. Secretaría Distrital de Salud de Bogotá, D.C. Anuario de vigilancia en salud pública, 2001. Boletín Epidemiológico Distrital 2002;7:4.

14. Martínez R, Jiménez $M$, León $\mathrm{CI}$, De la $\mathrm{Hoz} F$, Guerrero M. Prevalencia de VIH, tuberculosis y coinfección en sintomáticos respiratorios. Biomédica 1996;16(Sup.1):25.

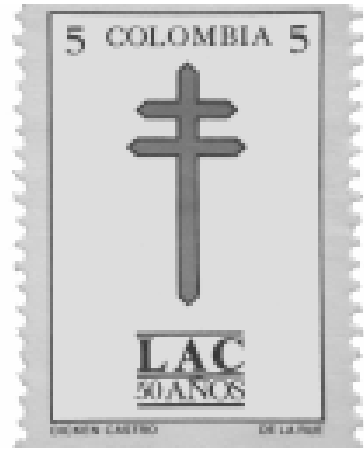

